\title{
Practical aspects when imaging using multiples with the Kirchhoff method
}

\author{
Pål Kristiansen, Frederico Xavier de Melo*, Dave Nichols, Schlumberger \\ Copyright 2019, SBGf - Sociedade Brasileira de Geofísica

\section{Primary versus multiple imaging}

This paper was prepared for presentation during the $16^{\text {th }}$ International Congress of the Brazilian Geophysical Society held in Rio de Janeiro, Brazil, 19-22 August 2019.

Contents of this paper were reviewed by the Technical Committee of the $16^{\text {th }}$ International Congress of the Brazilian Geophysical Society and do not necessarily represent any position of the SBGf, its officers or members. Electronic reproduction or storage of any part of this paper for commercial purposes without the written consent of the Brazilian Geophysical Society is prohibited.

\begin{abstract}
We discuss practical aspects when imaging multiples using the Kirchhoff Depth Migration method. Additional valuable information such as improving shallow imaging and getting wider illumination are possible if recorded multiples are properly extracted from the seismic data by type and order prior to the imaging process. We aim to provide a broader overview on methods to extract multiples by their signature type. We focus our analysis on simpler cases, such as first-order water-layer multiples, but the concept can be extended to any type of reverberation events, granted that a proper separation is put in place, so crosstalk is absent or significantly reduced.
\end{abstract}

\section{Introduction}

In surface seismic applications, multiples contain similar information about the reflectivity as the primaries but arriving at later times. When imaged as primaries, multiples generate crosstalk noise in the image, where careful attenuation or removal should be performed prior to conventional imaging. When correctly imaged, seismic multiples can overcome limitations in certain areas seen when imaging primaries. Significant effort has been done when working with multiples to solve imaging problems (Berkhout and Verschuur, 1994; Godfrey et al., 1998; Lu et al., 2011; Zhiping et al.,2015). To illustrate how multiples can be used in an imaging framework with comparable illumination with primaries,

Methods where the downgoing wavefield is injected as a secondary source to image multiples have been developed and used, particularly with multimeasurement streamer data (Lu et al., 2015; Ong et al., 2013). In addition to these wavefield injection methods, we developed methods that can image any order and type of multiple and work best when the different types and orders of multiples are separated (Lapilli et al., 2018). Separating primaries and multiples is done through prior processing, often improving the multiple attenuation outcome if we target different types and orders of multiples individually. The process is done by means of adaptive matching filtering techniques, as we also demonstrate that we can image the separated multiple using the methodology described herein.
Wide-tow acquisition configurations present shortcomings when properly recording primary data for shallow imaging. In such cases, the critical refraction of shallow events for the primary data comes at a short offset compared to the width of the streamer spread. On the other hand, multiples have smaller incident angles for the same offset, being better suited for shallow imaging along with a wider range of offsets.

Subsurface illumination is different for primaries than for multiples and varies between the different orders and types of multiples. Source-side multiples, for example, will provide wider illumination if compared with primaries because of the inherent sparsity coming from the source acquisition geometry. In such cases, this type of multiples can be used to fill in gaps between sail lines caused by sparse source distribution and with streamer feathering.

When imaging one order and type of multiple at a time, the multiple to image should be chosen based on which multiple best addresses the limitations observed in the primary imaging. Additional orders of multiples might be required to get the optimal image, and these are then imaged separately. The method presented here can image any multiple, given that they are extracted from the field records.

Consider a simple two-layer model used in Figure 1 simulating the reflection angle for primaries and first-order, water-layer, source-side multiple. Near offsets of $100 \mathrm{~m}$ and $350 \mathrm{~m}$ are used as typical near-offset values for inner and outer streamers, respectively.

The dotted part of the curves is where the reflection angle is above $50^{\circ}$. Note that the relation between reflection angle and offset is the same for the source- and receiverside multiples, which can both be imaged by the method described here.

\section{Multiple imaging using Kirchhoff depth migration}

Early work using Kirchhoff methods to image multiples demonstrated the process on sea-surface multiples for 2D deep-water, ocean-bottom, hydrophone data (Reiter et al., 1991). In the method described here, we use ray signatures in the ray tracing to accurately calculate traveltimes for a given multiple. The Green's function is then customized for the same set of reflections and transmissions that form the multiple being imaged.

Figure 2 shows an inline close to the middle streamer from a shallow-water survey. The image outcome in this case is rather similar and serves as a confirmation of the process of using multiples in migration algorithms. 


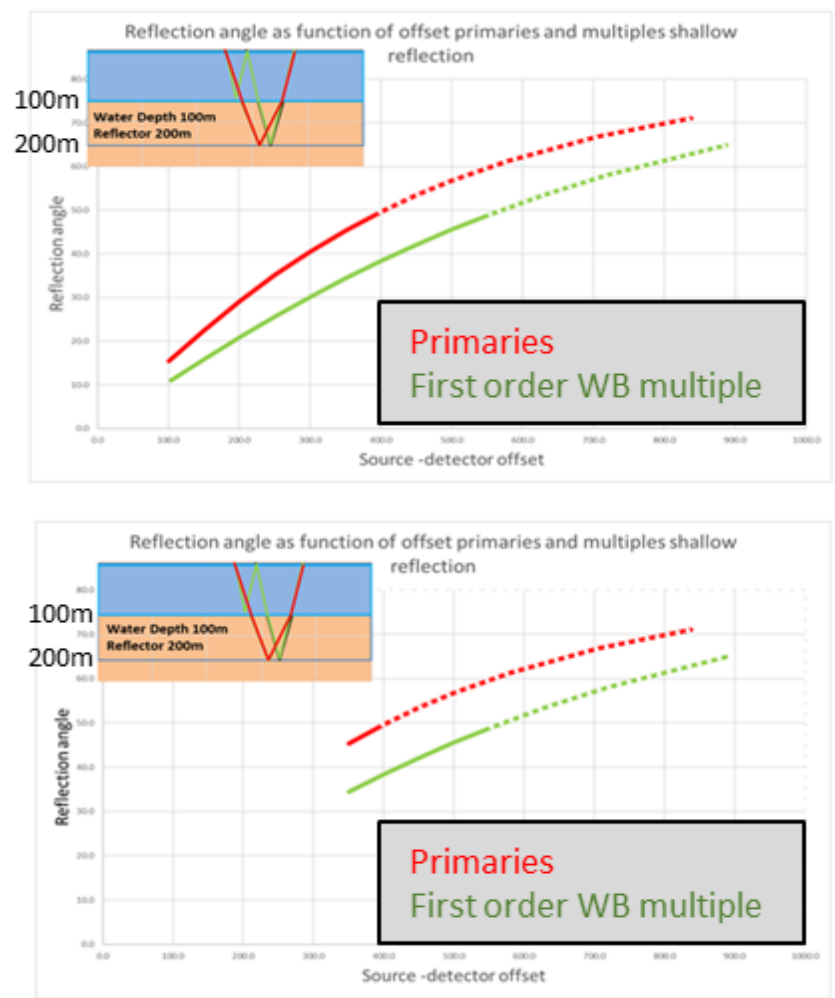

Figure 1 - Reflection angle as a function of offset for multiples (green curve) and primaries (red curve) for a reflector at twice the water depth. The display on top is for near-offset $100 \mathrm{~m}$ (inner streamer). The display below is for near-offsets $350 \mathrm{~m}$ (outer streamers). The dotted part of the curves is where the reflection angle is above $50^{\circ}$.

Since we are not relying on injecting a downgoing wavefield, both source- and receiver-side multiples can be imaged, as well as any non-free-surface multiple. Multiples are imaged using the multiple data that are extracted from the recorded seismic by type and order.

The method does not rely on separating up- and downgoing wavefields and uses the same earth model for imaging multiples as for primaries, as presented by Lu et al., 2015, and Ong et al., 2013). Compared with these methods, the proposed approach adds flexibility to the imaging multiples process, allowing us to define the best set of multiple raypaths to yield an improved illumination.

When imaging a given multiples by its type and order, it is generally required that we know a priori one of the formations where the surface multiple is being upward generated (used in the traveltime step). Offset gathers are output from the multiple imaging and can be used to pick residual moveout but can also be directly compared with primary offset gathers, preferably after converting to the angle domain. As only one type and order of multiple is imaged at a time, residual multiples of other types and orders will add noise to the multiple imaging.

\section{Separating multiples before imaging}

As the described method targets specific raypaths of multiples in the migration, events other than the expected in the imaging condition will add noise in the form of crosstalk. Therefore, it is important to have a method that is capable of separating multiples by their type and order to obtain an image free of crosstalk noise.

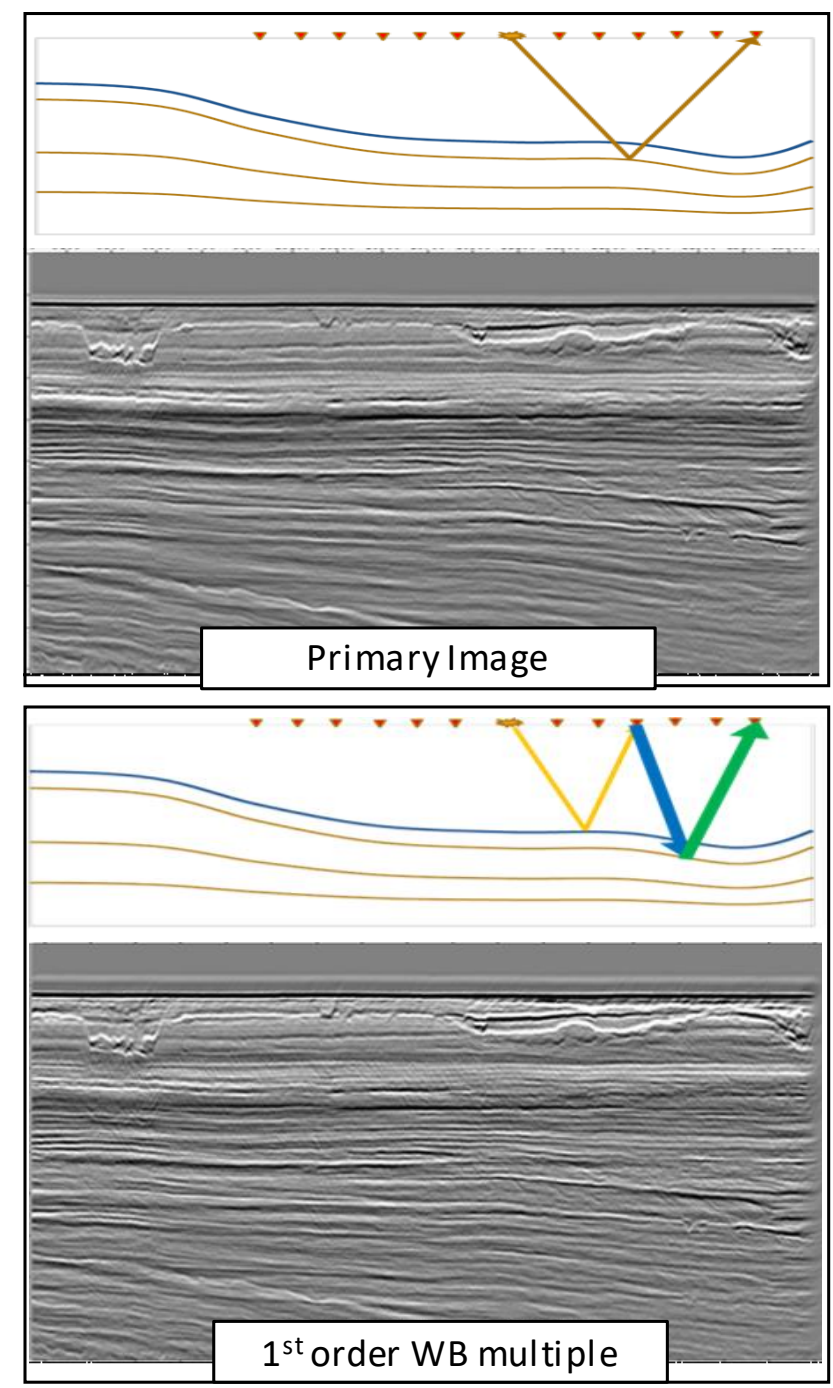

Figure 2- Schematic illustration with an inline image using primaries (top) and first water-layer source-side multiple (bottom). When the image is close to the streamer, both images have similar illumination.

Using the first-order water-layer-related surface multiples as an example, one way to extract this event from the recorded seismic data is by convolving an estimate of primaries $\widehat{P_{0}}$ (after complete multiple attenuation) with a Green's function representation of the water layer $G_{w b}$. This forward modeling can be performed for surface multiples with a bounce either at the source or at the receiver. The models are then matched with the seismic data to extract only the information pertinent to first-order water-layer surface-related multiples. Figure $3 \mathrm{a}$ shows a diagram of first-order water-layer-related multiples. 
Primary estimates can be accurately derived with highfidelity 3D shallow-water multiple attenuation techniques, such as the one described by Xavier de Melo et al. (2016). This same approach can also be extended to extract accurate models of source- and receiver-side water-layerrelated surface multiples, which are used to separate the recorded multiples in a least-squares sense. Separating these multiples allows imaging to either recover near-angle energy (receiver-side multiples) or to extend coverage (source-side multiples).

Other types and orders of multiples are separated using appropriate multiple attenuation techniques, assuming that the complexity of the separation process increases due to the multiplicity of possible events that can be associated with higher-order multiples. Figure $3 b$ shows an example of second-order water-layer related multiples that must be individually extracted from the seismic record for imaging.
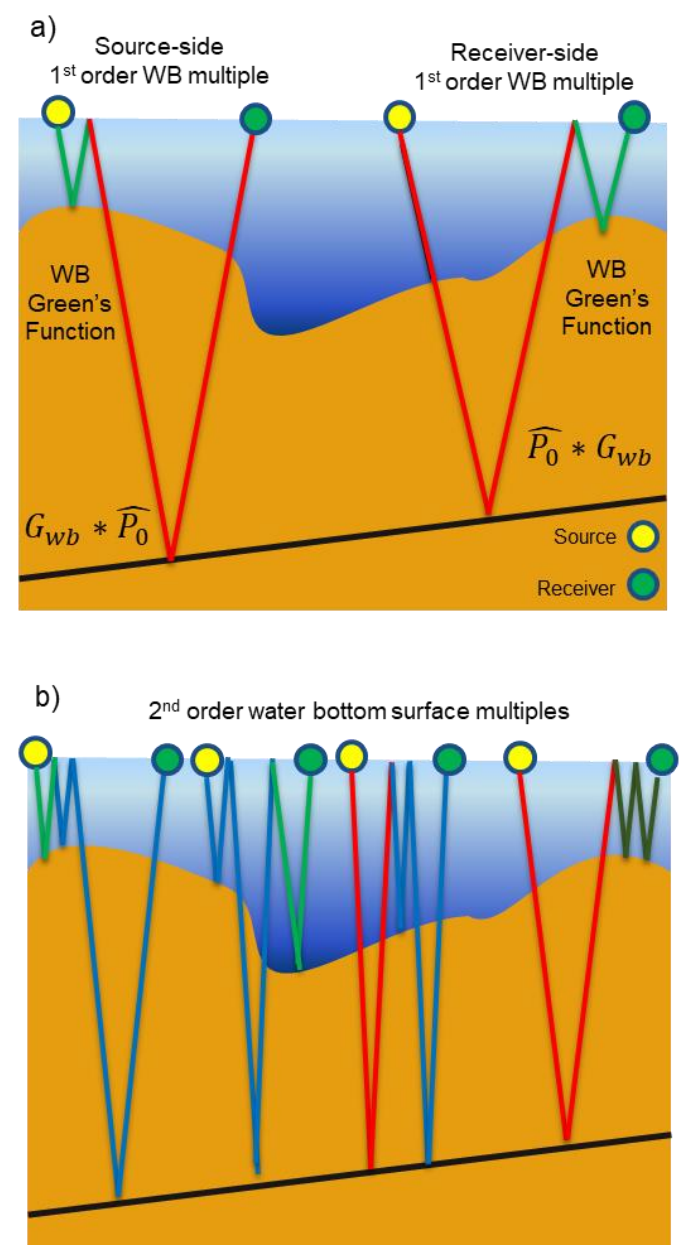

Figure 3 - Simple raypath diagram showing the possible types and orders of multiples that could be modeled and extracted for imaging. (a) First-order water-layer multiple can be simply divided into source- and receiver- side, allowing a straightforward workflow to extract it from the dataset. (b) Extending the problem to second-order multiples shows that the task of extracting specific signatures is possible, but significantly complex.

\section{Multiple imaging in shallow-water environment}

We used the proposed approach on a multimeasurement towed-streamer survey from the Bruce field area in the U.K. Continental Shelf sector of the North Sea, approximately $340 \mathrm{~km}$ northeast of Aberdeen. The survey sits in a relatively shallow environment, with an average water depth of $120 \mathrm{~m}$. Acquisition was performed using eight streamers, $3 \mathrm{~km}$ long each, separated by $75 \mathrm{~m}$ in the crossline direction and towed at an 18-m depth. The nearest receivers are placed $150 \mathrm{~m}$ from the source array. The multileveled source had subarrays at two different depths (6-9-6 m) to suppress source ghosts. Receiver deghosted shot gathers were reconstructed and equally spaced at $6.25 \mathrm{~m}$ between channels (inline) and cables (crossline).

When comparing migrated primary and multiple gathers as in Figure 4, we can clearly see the additional useful offsets in the shallow section from the imaged multiples. This not only gives a better shallow image, it also helps refine the shallow velocity model.

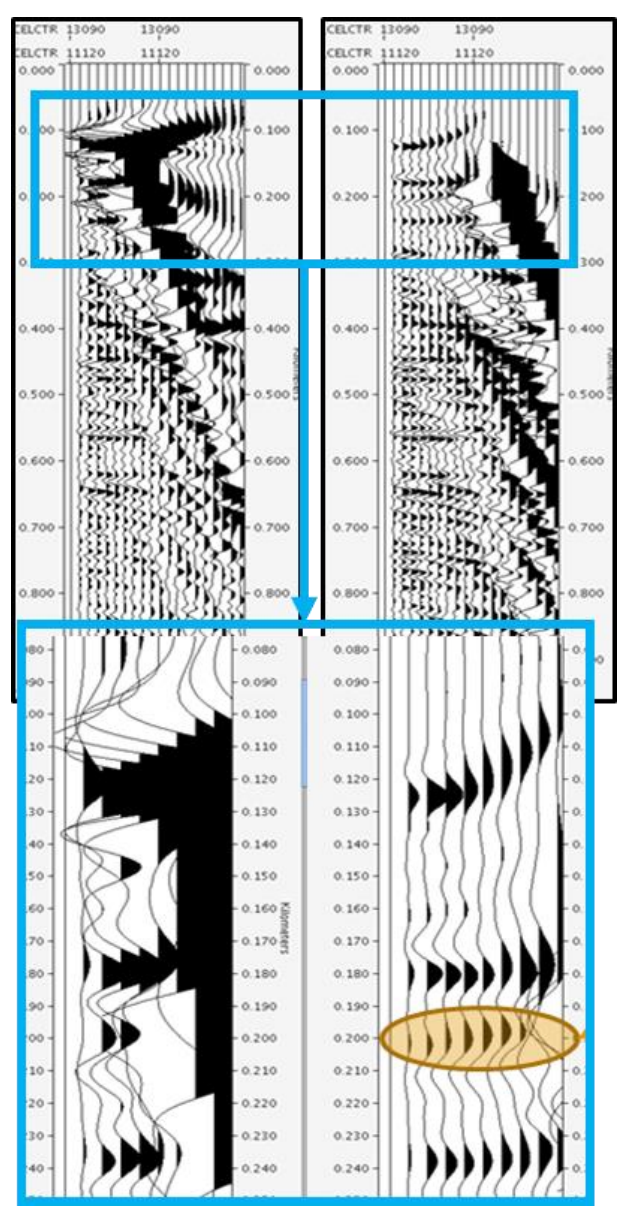

Figure 4 - Migrated primary and multiple gathers from a North Sea survey. Primary gather (top left), multiple gather (top right), zoomed-in primary gather (bottom left), and zoomed-in multiple gather (bottom right). On the zoomedin multiple gather, we indicate where residual moveout could be picked for a velocity model update. (From Kristiansen et al., 2018) 
Using the same model as above, Figure 5 shows an illustration of how primaries and multiples illuminate the subsurface. The example shows a 3D (crossline, timeslice, and inline) comparison between primary and sourceside multiples images. It is possible to see the shallow water infill improvement brought by source-side multiple in the crossline image, considerably reducing the acquisition footprint. The time slice at $116 \mathrm{~m}$ depth shows a better resolution in the source-side multiple image, the image using primaries is dominated by strong footprint artifacts. Taking on inline located between two sail lines, the shallow zone in the primary image shows a fainted near-seafloor response; whereas the image obtained from the sourceside multiples does not observe such behavior. In fact, the image resultant from the source-side multiple presents a better vertical resolution between for the first $250 \mathrm{~m}$. The similarity between primary and source-side multiple images beyond that also shows little evidence of crosstalk artifacts when the extraction of multiples from the seismic records is done properly.

\section{Conclusions}

We discussed a new approach based on the properties of a Kirchhoff depth migration method that is capable of imaging multiples based on a specific raypath signature. The method has the flexibility to image any given multiple signature, so long it is extracted by its type and order. We understand the proposed approach to be a complementary alternative to other imaging methods, including (but not limited) primary-only algorithms.

The proposed method for separating multiples is also capable of modeling and separating higher orders of waterlayer-related surface multiples, and just requires more modeling and matching iterations to achieve the desired multiple raypath. As the multiple order increases, possible raypath options the user wishes to separate also rises, along with the complexity of extracting these multiples from the field seismic record.

\section{Acknowledgments}

We thank WesternGeco Multiclient for permission to use their data and Schlumberger management for permission to present.

\section{References}

BERKHOUT A.J., and VERSCHUUR D.J., Multiple technology: Part 2, migration of multiple reflections: 64 th Annual International Meeting, SEG, Expanded Abstracts, 1497-1500, 1994.

GODFREY R. J., KRISTIANSEN P., ARMSTRONG B., COOPER M. and THOROGOOD E., Imaging the Foinaven Ghost: 68th Annual International Meeting, SEG, Expanded Abstracts, 1333-1335, 1998.
KRISTIANSEN P, XAVIER DE MELO F., and NICHOLS D., Multiple Imaging using the Kirchhoff method, 88th Annual International Meeting, SEG, Expanded Abstracts, 4156-4160, 2018.

LAPILLI C., PERDOMO J., and KOSTOV C., Imaging Free-surface Multiples Free of Noise: Fit-for-purpose Processing Methods and Application Examples, 80th EAGE Conference and Exhibition, Extended Abstracts, 2018.

LU S., WHITMORE D., VALENCIANO A.A. and CHEMINGUI N., Imaging of primaries and multiples with the 3D SEAM synthetic: 81st Annual International Meeting, SEG, Expanded Abstracts, 3271-3221, 2011.

LU S., WHITMORE D., VALENCIANO A.A. and CHEMINGUI N., Separated-wavefield imaging using primary and multiple energy: The Leading Edge 34, 770778, 2015.ONG C., LAPILLI C., PERDOMO J. and COATES R., Extended imaging and illumination in wave migrations: 83rd Annual International Meeting, SEG, Expanded Abstracts, 4116-4120, 2013.

REITER E.C., TOKSÖZ M.N., KEHO T.H. and PURDY G.M. Imaging with deep-water multiples: Geophysics, 56(7), 1081-1086, 1991.

XAVIER DE MELO F., KOSTOV C. and COOKE A., Shallow-water Free-surface Multiple Attenuation on Multimeasurement Data - A Case Study from the North Sea: 78th EAGE Conference and Exhibition, Extended Abstracts, 2016

ZHIPING Y., JESHURUN H., CHEN H., and YANG J., Reverse time migration of multiples: Applications and challenges: The Leading Edge, 34, 780-786, 2015. 

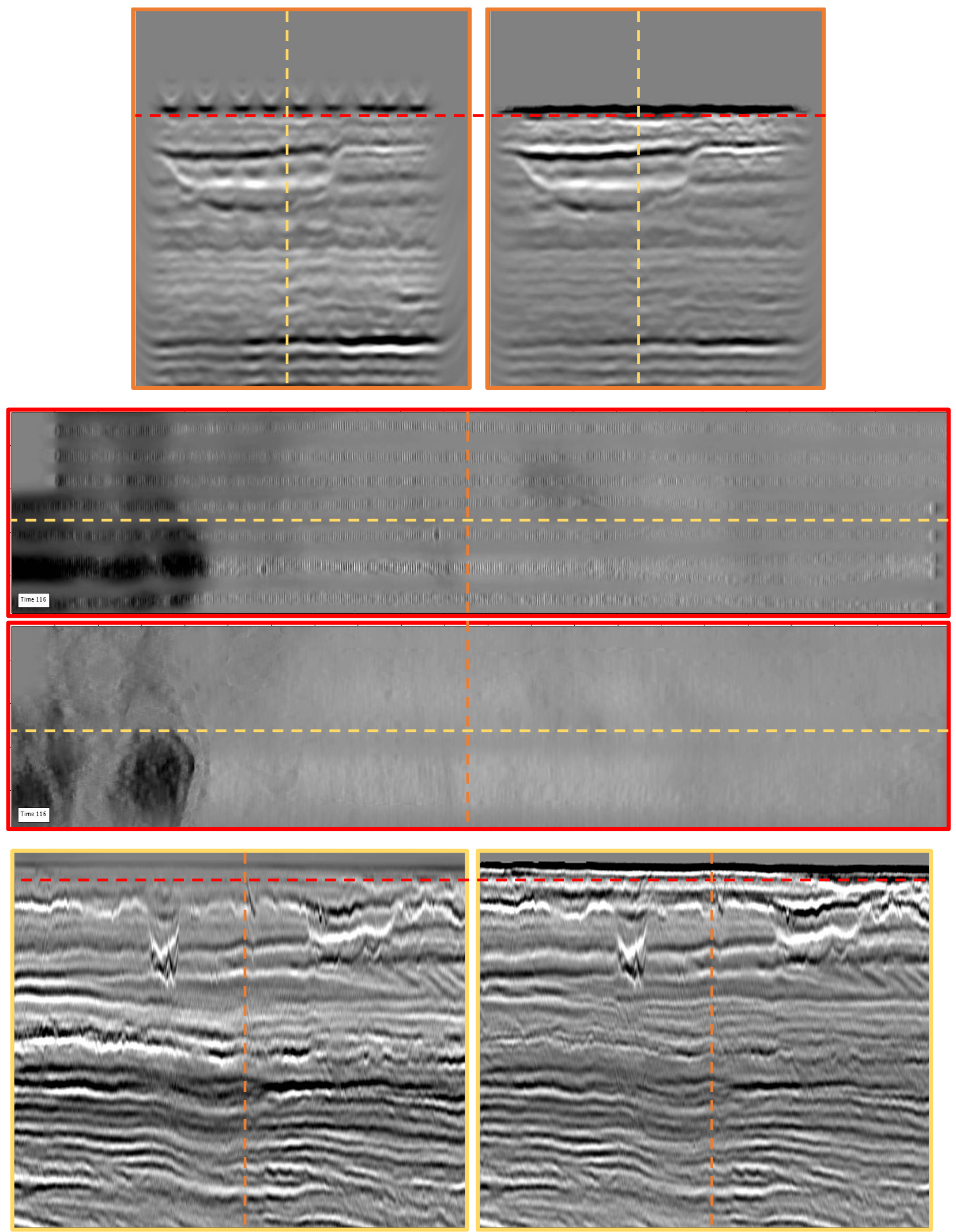

Figure 5 - Crossline (top), time-slice at $116 \mathrm{~m}$ (middle) and inline (bottom) comparison between images of primaries and firstorder source-side water-layer multiple. Examples clearly shows the infill and near-seafloor resolution improvement when using the source-side multiples. The deepest section of the crossline and inline images also show that primary and multiple-based methods have similar response, meaning that the extraction of seismic multiples were successful. 